\section{$010-011$ \\ Noticias y comentarios \\ PH47 - Febrero 2004}

Enrique Morales lamentó que el desconocimiento por parte de los técnicos de las características de la madera les lleve a desconfiar de su comportamiento, indicando que sólo se acude a ella cuando hay que restaurar un edificio histórico construido en madera o realizar un edificio singular, por lo que su consumo está muy por debajo de Estados Unidos, Suecia, Finlandia, Canadá o Chile. Igualmente repasó las características técnicas y los requisitos de empleo y conservación, sintiendo que en demasiadas ocasiones los elementos de madera se hayan sustituido por otros materiales en la restauración de monumentos andaluces.

Finalmente, José María Cabeza -al referirse a la cerámica- señaló cómo en los últimos años el número de intervenciones en edificios con decoración cerámica se ha incrementado, si bien en muchos casos no se ha tenido en cuenta la necesidad de estudios previos del material y del modo de afrontar la restauración del revestimiento cerámico por parte de especialistas en el tema. Esto se debe, según dijo y entre otros fac- tores, a la "visión" de la cerámica aplicada en la arquitectura como un bien menor o de segundo orden, considerando la obra arquitectónica como un mero contenedor espacial al margen de los elementos que la enriquecen y le confieren su carácter global. Hoy, no obstante, indicó, tenemos que reconocer que la cerámica se encuentra en pleno apogeo, consecuencia entre otras razones, de la aplicación de las teorías del color en arquitectura. Igualmente recordó que la cerámica posee una gran importancia en la edificación existente por el valor etnográfico que aporta y la extraordinaria información que nos ofrece para identificar el pasado de nuestro importante patrimonio edificado.

Las Jornadas Técnicas sobre el Patrimonio que anualmente organiza la Fundación Aparejadores fueron seguidas en esta edición por numerosos profesionales y estudiantes de Arquitectura, Ingenieria, Bellas Artes, Química... que participaron, a su vez, en interesantes debates sobre los referidos materiales básicos, tras las conferencias impartidas por los expertos

\title{
La Asociación de Restauradores sin Fronteras hace balance de las intervenciones sobre metales
}

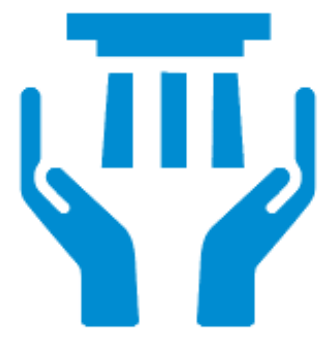

El II Seminario Internacional organizado por la Asociación Restauradores Sin Fronteras se celebró en el Institut Royal du Patrimoine Artistique IRPA de Bruselas, del 24 al 26 de octubre del 2003.

El seminario fue presentado por el moderador del encuentro, Regis Bertholon (Universidad de Paris I, Sección Conservación-Restauración de Bienes Culturales), y la directora en funciones del IRPA, Myriam Serck-Dewaide.

Siguiendo el orden cronológico del programa, las primeras conferencias fueron sobre temas arqueológicos. Sylviane Mathieu, del Ministerio de la Región Valona de Bélgica, nos presentó unos casos prácticos donde se ilustraban los problemas que aparecen en cuanto a la gestión de piezas arqueológicas después de su descubrimiento en la excavación. Después, Stephane Pennec, especialista francés, quiso mostrar el caso de la restauración de una reja-celosía en el centro histórico del Cairo, una referencia para los equipos que trabajan en situaciones similares. Seguidamente, el Dr. Joaquín Barrio de la Universidad Autónoma de Madrid hizo hincapié en la conservación a largo plazo de las piezas arqueológicas, poniendo como ejemplo piezas prerromanas encontradas en excavaciones en España.

Pasando a la época prebizantina, la Dra. María Jesús Sánchez, Jefa de Sección de Materiales de Arqueología, Etnología y Artes Decorativas del Instituto de Patrimonio Histórico Español, expuso el estudio metalúrgico de la magnífica pieza de la Real Academia de la Historia de Madrid, el Missorium de Teodosio. Antonio Tosini, conservador-restaurador del Museo de Capodimonte en Nápoles, explicó las características de dos armas de fuego de Carlos III de Borbón. Gracias a los trabajos de restauración realizados se descubrió la autoría y la importancia de estas armas pertenecientes a la colección del Museo. Giancarlo Marini escultor y conservador-restaurador autónomo establecido en Florencia mostró, gracias a sus investigaciones y sus conocimientos técnicos de escultor, los descubrimientos realizados sobre la técnica de fundición de esculturas en bronce de grandes dimensiones. La comunicación de Paul Tear de The Wallace Collection de Londres nos definió los sistemas de limpieza, cómo elegir los productos adecuados para la eliminación de la oxidación y, finalmente, si para su protección es necesario barnizar los bronces dorados.

Al dia siguiente, el programa de comunicaciones continuó con los estudios e intervenciones del patrimonio más próximo a nuestros días y las últimas investigaciones científicas en la materia. La Dra. Immaculada Aguilar, de la Universitat de València, habló sobre el puente de hierro en la Comunidad Valenciana y la fragilidad de este patrimonio que está desapareciendo. Más tarde Lydia Beerkens, coordinadora de la Sección de Arte Moderno de la Escuela Instituto de Maastricht (Paises Bajos), propuso una participación conjunta de conservadores-restauradores de metal y los especialistas en conservación de 
arte contemporáneo, exponiendo a su vez la problemática que conlleva la conservación de este tipo de piezas. Virginia Costa, representando al Laboratorio de Investigación de los Monumentos Históricos franceses (LRMH), repasó las técnicas electroquímicas aplicadas a la conservación de metales y como modificar el método para un uso más adecuado.

En representación de Rocco Mazzeo (Universidad de Bolonia), Paola Letardi, del Instituto de Ciencias Marinas de Génova, propuso una mayor investigación de los problemas de corrosión de bronces expuestos al exterior. La sección de Metales de la Escuela Superior de Amberes, dirigida por Patrick Storme, mantiene una politica de investigación que mostró en el seminario como modelo de programa de formación en conservaciónrestauración de metal. El Dr. Christian Degrigny, coordinador del grupo de trabajo Metal del ICOMComité para la Conservación, comentó el dinamismo y el esfuerzo que el Consejo Internacional de Museos hace para conseguir más dialogo. En el espacio reservado para A-RSF se presentó la primera parte de las actividades de la asociación en Costa Rica, el Proyecto Alajuela, que se llevó a cabo en el verano 2003. En dicho proyecto participaron Montse Artigau, profesora de restauración de materiales inorgánicos en la Escuela de Conservación y Restauración de BB CC de Barcelona, y Margarida Silva, restauradora-conservadora, quienes presentaron el trabajo realizado: restauración e investigación de una fuente de metal del siglo XIX.

Finalmente, la mesa redonda, moderada por Regis Bertholon y Juan Carlos Bermejo, se dirigió hacia dos vertientes: el patrimonio industrial y la corrosión de metales expuestos al aire libre, debate que contó con la presencia de Herman
Terryn de la VUB (Universidad Libre de Bruselas), quien habló de casos tan conocidos como el estudio del Atomium; de Immaculada Aguilar, que habló del Programa de Conservación del Patrimonio Industrial Español y de la necesidad de la catalogación para la conservación de todos los elementos del patrimonio industrial, y de Myriam Serck-Dewaide, quien propuso una colaboración entre los especialistas de patrimonio industrial y el IRPA, para poder avanzar en la investigación.

El tercero y último día, se hizo una visita al Museo de Arte Antiguo de Namur para conocer la exposición "Alrededor de Hugo d 'Oignies", artista que realizó las piezas de orfebrería mosana pertenecientes al Tesoro del Antiguo Priorato de San Nicolás, fundado a principios del siglo XIII en Oignies, cerca de Charleroi, Bélgica.

El encuentro se caracterizó por la buena atmósfera, la calidad de las comunicaciones y la participación constante del público. Como muestra de los buenos resultados, se anunció el III Seminario Internacional A-RSF, que se celebrará en Valencia en el 2005, esta vez bajo el tema de Conservación de Interiores Históricos.

Nos gustaría agradecer a Regis Bertholon su ayuda durante todo el seminario y a Myriam Serck-Dewaide por poner a disposición de la Asociación Restauradores sin Fronteras las instalaciones del IRPA.

Juan Carlos Bermejo Cejudo y Raquel Ballester García

Coordinadores del II Seminario

Internacional A-RSF

\section{El Algarve y Andalucia dialogan sobre patrimonio y museología}

Las VII Jornadas de Patrimonio del Algarve, organizadas inicialmente por la Cámara Municipal de Albufeira y, desde hace dos años, en colaboración con la Universidad de Algarve, se celebraron los días 23 y 24 de octubre de 2003 en Albufeira.

Hacer un balance sobre el desarrollo de las VII Jornadas pasa por destacar en primer término el diálogo que se estableció entre el Algarve y Andalucia, dos regiones limítrofes que a pesar de su cercanía no encuentran frecuentemente escenarios donde exponer experiencias paralelas. Así, podríamos extraer como puntos de conclusión, que serán detallados en la publicación de las Actas de las Jornadas que se está gestionando, la buena iniciativa de hacer extensivo el tema de las jornadas -la Museología- a nuestro país, acor- tar distancias (que realmente en cuanto al modo de trabajar no son tales) y destacar el interés por el acercamiento a nuestra realidad patrimonial, que también deberíamos no sólo valorar sino fomentar desde nuestras posibilidades por la riqueza que pueda aportarnos a todos.

Hay que valorar este interés de la comunidad portuguesa por aproximarse a España, a Andalucía, por intercambiar y poder confluir en proyectos comunes, especialmente en el ámbito de la educación en los museos.

$M^{\mathrm{a}}$ Teresa Suárez Domínguez Historiadora del Arte, especializada en Museos y Patrimonio 